\title{
Type la supernovas and fusion of black holes: Do they complement each other in measuring the expansion of the universe?
}

\section{Rafael Girola-Schneider ${ }^{\mathbf{1}}$}

Universidad Nacional de Tres de Febrero, Buenos Aires, Argentina.

E-mail: rafaelgirola@yahoo.com.ar

This work aims to show if it is possible to perform distance measurements, complementing type Ia supernovae with the fusion of black holes.

It will be evaluated if it is possible to refine the measurement of the accelerated expansion of the universe, through the detection of gravitational waves generated by the fusion of black holes and comparing them with the intrinsic luminosity of type Ia supernovae.

International Conference on Black Holes as Cosmic Batteries: UHECRs and Multimessenger Astronomy - BHCB2018

12-15 September, 2018

Foz do Iguaçu, Brasil

${ }^{1}$ Speaker 


\section{Introduction}

The models of the universe with dark energy are characterized by the macroscopic effects in the history of the expansion of the universe and therefore in the formation of large structures. The main characteristic of these scenarios is dark energy, responsible for the acceleration in the expansion of the universe and gravity. The result is that the combination of both, progressively structures the universe by building large voids, filaments, supercumuli and galaxy clusters [9] [12].

The evaluation of the diagnosis on the nature of dark energy is necessary to know its footprint in astronomical observations, called cosmological soundings. This is the case of type Ia supernovae that reveals the effects of the expansion and geometric property of the universe.

We estimate that the gravitational waves generated by the fusion of black holes could also reveal the effects of the expansion and its geometric properties. Therefore for not having a sufficient population of gravitational wave sources from the fusion of stellar black holes, we took a population of type Ia supernovae and we contrasted the results assuming the fusion of black holes in the places where they have been observed and measured. The expansion rate according to type Ia supernovae taking the measured data from the observed sources of the black hole fusion. Then, we wonder if, by means of a theoretical population of gravitational wave sources from the concrete data of certain measured sources, in the same places where the type Ia supernova events have occurred, the measurements are concordant or not [10].

According to the sensitivity and quantity of black hole mergers, one can calculate the rate at which black holes form in the universe. It is expected between 3 and 90 significant detections of mergers of black holes during a period of observation and $100 \%$ of detecting at least one. Meanwhile, it is simulated with a theoretical population of black hole mergers for testing.

During the last decade, several groups of researchers led by astronomers Alan Riess and Saul Perlmutter [8] have been using type Ia supernovas as standard candles. A standard candle is only a light source whose intrinsic brightness is known and used to measure distances. The enormous utility of the supernovas resides in the fact of being able to rival in brightness with the group of stars of their galaxy of origin, and therefore to be one of the ways to know the distance of the more distant galaxies. The results show that supernovas at a given redshift are less bright than expected in a universe of low density. It could mean that the universe is expanding rapidly. Thus, the expansion was slower in the past, so the universe must have needed more time to reach the current separation of objects and therefore the light of the supernova must have taken more time to reach us, which implies a greater apparent distance and consequently a lower apparent brightness.

From the observations of type Ia supernovae, it was determined that, in effect, the Universe is in accelerated expansion [7]. Subsequently it has been possible to reach the same conclusion and very similar numbers using other techniques, such as studying the "baryon acoustic oscillations" and the structure and distribution of galaxy clusters in the large-scale structure of the Universe. However, what would happen if a factor that has not been considered varies the maximum luminosity of a type Ia supernova? From the theoretical point of view, this luminosity also depends on the amount of nickel $\left({ }^{56} \mathrm{Ni}\right)$ synthesized during the supernova 
explosion, which in turn depends on the amount of carbon $(\mathrm{C})$, oxygen $(\mathrm{O})$, nitrogen $(\mathrm{N})$ and iron $(\mathrm{Fe})$ of the white dwarf.

Thus, several theoretical studies suggest that, in effect, the maximum brightness of a type Ia supernova is not absolute, but also depends on the chemical composition of the star system. White dwarfs richer in metals will suffer from less bright supernova explosions than white dwarfs that have less metal content. Or, at least, the theory says so.

How can we demonstrate this? Through observations of supernovas in galaxies where we can establish the content in metals. If we can measure on the one hand the "metallicity" of a galaxy where a type Ia supernova has exploded and its distance (using a method independent of supernovas for it) we could observe if such dependence predicted by the theoretical models exists. This was the idea that the theoretical astrophysician Mercedes Mollá (CIEMAT, Madrid) had back in 2009. The motivation of her work was related to the work done by Manuel MorenoRaya [6], from the University of Granada (Spain), who found the following: based on a final sample of 28 galaxies and despite the dispersion of data and the errors in the measurements, a statistical analysis revealed that, with an $80 \%$ probability, that there is a relationship between the two measurements, so that type Ia supernovae with less amount of metals have higher absolute brightness, and type Ia supernovae with less metal have higher absolute brightness. The data point out that the non-metallic type Ia supernovae shine on average 0.14 more magnitudes than the more metallic type Ia supernovae (see Fig. 1) [5].

As these drawbacks of type Ia supernovae do not affect cosmological models in themselves, in particular the accelerated expansion model, we propose to measure the accelerated expansion of the universe by the detection of gravitational waves complemented by type Ia supernovae. 


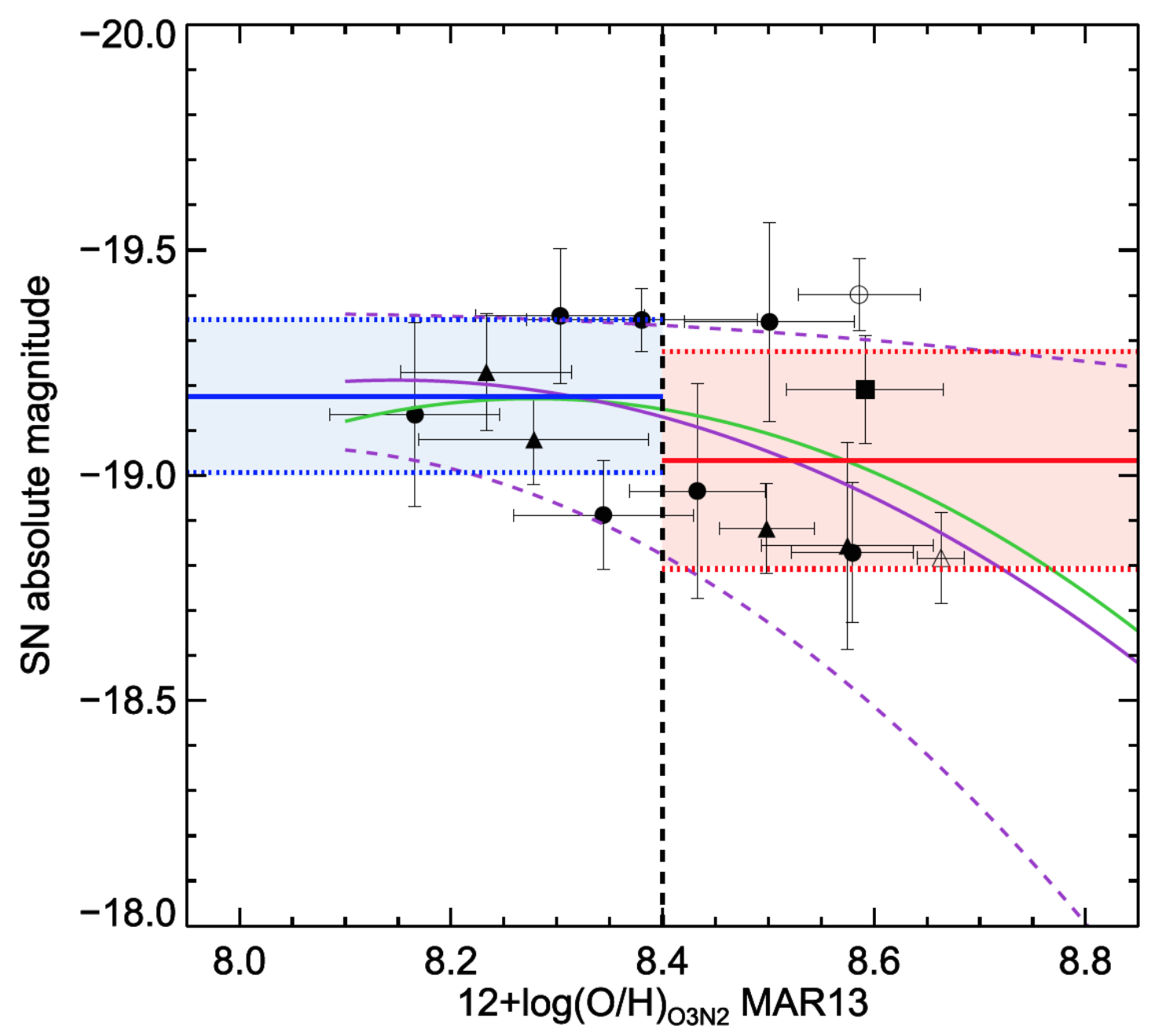

Fig.1: This diagram shows the absolute magnitude of type Ia supernovae (vertical axis) as a function of oxygen abundance (horizontal axis) given by "empirical calibrations". Oxygen abundance is used to measure the "metallicity" or metal content of a galaxy with star formation. The methods to determine the distance to the galaxies are given by triangles (using the Tully-Fisher relation), luminosity function of planetary nebulae (squares) and combining the results of Cepheid variable stars with Tully-Fisher relation (circles). The green line is a second-order adjustment to the data. The purple line is an adjustment assuming four averages in metallicity. The blue and red horizontal lines represent the mean value at low and high metallicity, respectively, with their error. Figure extracted from [6].

\section{Preliminary work}

We developed an algorithm based on the Benchmark model using the data of GW 170814 [1], GW 170608 and GW 170817 [4,11] and the following type Ia supernovae located in the indicated host galaxy respectively: 1937C (IC4182), 1895B (NGC 5253), 1972E (NGC 5253), 1981B (NGC 4536), 1960F (NGC 4639) and 1989B (NGC 3627). For this we assume fusion of black holes of equal masses instead of type Ia supernovae and then we obtained the distance. For example: GW170817 represents the first time that gravitational and electromagnetic waves from the same astrophysical source have been observed. This joint observation supports the hypothesis that the source comes from the fusion of two neutron stars, which has also allowed the identification of the host galaxy, a galaxy known as NGC 4993 in the constellation of Hydra. 
Trough the analysis of the gravitational wave GW170817, we could estimate the distance in approximately $44 \mathrm{Mpc}$, based on the hypothesis that the position of GW170817 is exactly coincident with NGC 4993 [10].

The distance obtained has an uncertainty of 15\%, resulting from the combination of instrumental noise in the detectors and the fact that we do not know precisely the inclination of the plane of the orbit of the neutron binary star with respect to the Earth. To estimate $\mathrm{H}_{0}$ it is necessary to combine the distance to NGC 4993 obtained by gravitational waves with the radial velocity of the galaxy due to the Hubble expansion. However, we can not simply measure the radial velocity of NGC 1993 since the expansion of the universe is not smooth and uniform in all its points: the granulation of the universe modifies Hubble's expansion in small scales since the galaxies have their own movements (known as "peculiar velocities") caused by the gravitational attraction of other galaxies and galaxy clusters (and also by the invisible dark matter) in their surroundings. The relative closeness of NGC 4993 implies that the value of its peculiar velocity is probably a significant part of its measured radial velocity and, therefore, can not be ignored.

This work consists in contrasting the measurements made by type Ia supernovae in different redshifts and involves the measurement of the acceleration of the universe with gravitational waves. Assuming a distribution of binary black hole systems in places where type Ia supernovae have been detected, we measured the distances in the different redshift by detecting the simulated gravitational waves: Do distances differ? Do the calibrations to value $\mathrm{H}_{0}$ improve?

\section{Gravitational waves: Is its detection possible to be used as cosmological probes?}

The supernovae have the particularity of their luminosity curves and their absolute luminosity at their maximum. Of course, there are error limits depending on the environment in which the event is generated. The difference of apparent luminosity is not more than a simple effect of distance that depends on the expansion, and it allows the measuring of acceleration and the effects of dark energy. The luminosity of type Ia supernovae allows us to observe hundreds of them distributed from our galaxy in distances greater than 10 billion light-years. There are two major categories of tests to measure the expansion of the universe: effects of the expansion and geometric properties of the universe and those that reveal the effects of gravitation and the structure of the universe. Hence type Ia supernovae belong to the first category. If we know the maximum luminosity of the type Ia supernova, it is possible to convert its mass, and if we know the masses of the sources that originate gravitational waves, it is possible to perform the symmetric conversion to estimate and limit the error measurements of type Ia supernovae assuming in the places where supernovae have been detected, a source of gravitational waves. Then the gravitational waves could also be supernovae Ia type proofs [4,9].

\section{Bayesian inference}

By Bayesian inference, taking as a reference the host galaxy of the fusion of neutron stars and its derivation in gravitational waves, we obtain a list of host galaxies where type Ia 
supernovae have been registered. Bayesian reasoning provides a probabilistic approach to inference. It is based on the assumption that the amount of interest is governed by probability distributions and that optimal decisions can be made by reasoning about these probabilities together with the data obtained. This approach is being used in many fields of research, of which we can highlight mobile robotics and computational vision, both related to the content of this thesis. In this section we want to define two of the tools used in the development of this thesis: Bayes' theorem and the minimum description length principle.

As we know, the characteristic of gravitational waves is that they move at speed of light, and they are not interfered by the interstellar medium as (if) it occurs with electromagnetic waves, which originate in stellar collapses, supernovae, fusion of black holes, becoming beacons to map the universe in terms of the geometric characteristics and referents of the expansion of space. Therefore, we can see that they can be approximate dimensions with respect to other distance methods such as type Ia supernovae. Also in the collapse of a white dwarf there is generation of gravitational waves so that, depending on the source mass, it is possible to simulate the detection of gravitational waves.

\section{Gravitational wave source data}

The data that will be imported to perform the calculation are: type of source, time dme merger, distance and redshift and the total mass since given the mass we can estimate the equivalent to its brightness and estimate the magnitude: that is, according to the sources of type Ia supernovae, by means of the hypothetical fusion of black holes in the places where they have been detected, to estimate the mass, to estimate the luminosity then the magnitude and to use the relationship of stellar candles in a universe in acceleration. We need the equation that relates the luminosity distance with $\mathrm{H}_{0}$ and the deceleration parameter $\mathrm{q}_{0}$ and $\mathrm{z}$ the redshift in the Benchmark model and compare with the distance modulus $\mathrm{m}-\mathrm{M}$.

For example with the data of GW 170608, the source is the fusion of black holes estimating a distance of 0.7-1.5 billion light-years with a redshift 0.04-0.1 and a total mass of 18-24 solar mass. This information by means of a Bayesian algorithm allow us to estimate the response of virtual gravitational waves in the observed places of type Ia supernovae, transforming the data of gravitational wave sources according to the mass transformed in the gravitational wave energy. Then we convert it in the equivalent of luminosity and from there, we recreate it with the data of the supernova models and we analyze the contrasts [7].

\section{Conversion process}

The masses of the binary system are estimated: the mass transformed into energy of the gravitational wave is estimated. The propagation speed is c. Referential data of observed mergers are taken. An algorithm that contains the information from a population of type Ia supernovae estimating fusions of black holes with the known data is modeled and performed. The conversion of the energy of the gravitational wave into the corresponding mass is carried out, then its luminosity equivalence and the photometric equation $m=-2.5 \log \left(\phi / \phi_{\mathrm{x}}\right)$ is used 
where $\phi_{\mathrm{x}}=253 \times 10^{-8} \mathrm{~W} \mathrm{~m}^{-2}$. Then the conversions of magnitudes are made and related to the luminosity, the absolute magnitude: $\mathrm{M}=-2.5 \log \left(\mathrm{L} / \mathrm{L}_{\mathrm{x}}\right)$ where $\mathrm{L}_{\mathrm{x}}=78.7 \mathrm{~L}_{0}$ [3].

The figure 2 shows the isochrones of constant $\mathrm{H}_{0} \mathrm{t}_{0}$. the age of the universe in units of the Hubble time $\mathrm{H}_{-10}$, with the best-fit $68 \%$ and $90 \%$ confidence regions in the $\left(\Omega_{\mathrm{M}}, \Omega_{\Lambda}\right)$ plane.

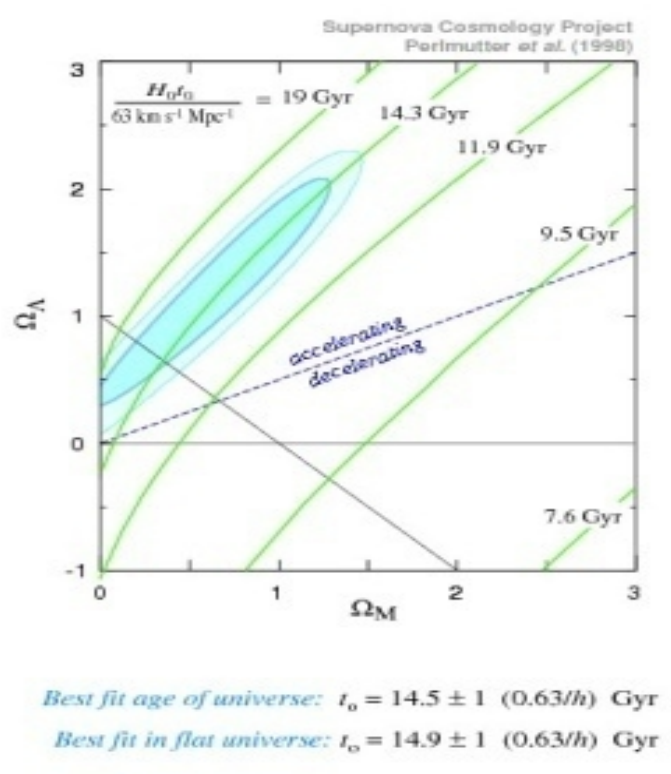

Fig.2: Isochrones of constant $H_{0} t_{0}$, the age of the universe relative to the Hubble time $\mathrm{H}_{0}$, with the best-fit $68 \%$ and $90 \%$ confidence regions in the $\Omega_{M}-\Omega_{\Lambda}$ plane for the primary analysis. Figure extracted of [8].

The objective of the work is given a population of gravitational waves, whose origin is the fusion of black holes, the result of them is consistent with the graph, using them as "candles" to evaluate $\mathrm{H}_{0}$, and hence the cosmological parameters.

To estimate in a first approximation the contrast between distance indicators with type Ia supernovae and gravitational waves generated by the fusion of black holes, in the equivalent places where there is data of the supernovas and their distances, the fusion of black holes will be taken as data (black holes already observed and gravitational waves recorded). At first, the objective is to measure the mass that is transformed into energy associated with the gravitational wave and estimate according to the equivalent mass, which luminosity would correspond to the equation that relates the absolute magnitude $M$ with the distance. That is, simulating a population of "star candles" of luminosity equivalent to the equivalent mass of the energy associated with the gravitational wave. Then, to measure the redshift $z$ and the flow $\phi$ for each candel by computing $d_{L}=(L / 4 \pi \phi)^{1 / 2}$. Given the environment where the fusion of black holes is observed and knowing the distance of the environment of the source by some particular method, for example if it is a spiral galaxy by the Tully-Fisher method, we contrast it with the type Ia supernova method and eventually with the gravitational waves. The question is whether there is convergence, or a margin of error that makes the results compatible [2,5].

For the assumed mergers with $z=0.2$ we use the Benchmark model $q_{0}=-0.53$. With a luminosity distance $\mathrm{d}_{\mathrm{L}}>1000 \mathrm{Mpc}$, we use the type Ia supernova method: $\mathrm{L}=4 \times 10^{9} \mathrm{~L}_{0}$ and $\mathrm{z} \approx 1$ 
The module distance $m-M=5 \log 10\left(d_{L} / 1 M p c\right)+25$. Narrowing for $z<<1$ and with the data of the distance module and the distance luminosity to the Great Magellanic Cloud and the Virgo Cluster (18.5; 0.050 Mpc and 30.9; $15 \mathrm{Mpc}$, respectively, with $\mathrm{d}_{\mathrm{L}} \approx \mathrm{C} / \mathrm{H}_{0} z\left(1+\left(1-q_{0}\right) / 2 z\right)$ we want to contrast the data of the gravitational wave fusions with the following expression: $\mathrm{m}-\mathrm{M}$ $=43.23-5 \log 10\left(\mathrm{H}_{0} / 68 \mathrm{Km} \mathrm{s}^{-1} \mathrm{Mpc}^{-1}\right)+5 \log 10\left(z+1,089\left(1-q_{0}\right) z\right)$.

\section{Conclusions}

By means of the simulation of gravitational waves and assuming the fusion of black holes in the places where the distance from the source to the observer has been detected and measured, we try to contrast it with the distance measurement to improve errors given by the environmental conditions of the white dwarfs that generate supernovae type Ia. This work is preliminary where, by means of a Bayesian algorithm with the acquired data of the gravitational waves identified by LIGO (Laser Interferometer Gravitational Wave Observatory), it extends to a supposed population of gravitational waves to measure the expansion of the universe in different redshifts accusing the possible error of type Ia supernovae.

Gravitational waves can resolve the uncertainty in the measurement of extragalactic distances by type Ia supernovae, which can be taken as "candles". We understand that this is a controversial issue because of the few data still obtained, but it could further narrow the value of the Hubble constant provided by the data obtained through LIGO. Since the data is still scarce, It is expected an improvement of the measurements with the new data obtained by LIGO.

\section{References}

[1] B.P. Abbott et al., Phys. Rev. Lett., 2017, 119, 141101.

[2] R.J.A. Cambourne, Relativity Gravitation and Cosmology, Cambridge University Press (2010).

[3] J. García-Bellido, Astrophysics and Cosmology, 1999, Invited lecture at European School of High Energy Physics, Slovack Republic, arXiv:hep-ph/0004188.

[4] D.E. Holz \& S.A. Hughes, ApJ, 2005, 629,15.

[5] M.H. Jones, R.J.A. Lambourne \& S. Serjeant,An Introduction to Galaxies and Cosmology $2^{\text {nd }}$ edit., Cambridge Open University (2015).

[6] M.E. Moreno-Raya, M. Mollá, A.R. López-Sánchez, L. Galbany, J.M. Vílchez, A. Carnero Rosell, I. Domínguez, ApJ, 2016, 818, L19.

[7] J.C. Niemeyer \& J.W. Truran, Type Ia Supernovae, Theory and Cosmology, Cambridge Contemporany Astrophysics (2010).

[8] A. Riess, P. Nugent, A. Filippenko, R. Kirshner \& S. Perlmutter, ApJ, 1998, 504, 935.

[9] B. Ryden, Introduction to Cosmology $2^{\text {nd }}$ edit, Cambridge University Press (2017).

[10] R.H. Sanders, Deconstructing Cosmology, Cambridge University Press (2016).

[11] B. Schutz, Nature, 1986, 323, 310.

[12] J. Silk, Le future du cosmos, Odile Jacob Publie, France (2015). 